\title{
The Rumen Flagellate Callimastix frontalis: does Sequestration Occur?
}

\author{
By C. G. ORPIN \\ Department of Biochemistry, Agricultural Research Council, Institute of \\ Animal Physiology, Babraham, Cambridge CB2 $4 A T$
}

(Received I9 March 1974)

INTRODUCTION

During studies of the diurnal changes in the concentrations of micro-organisms in the rumen of sheep fed limited diets once daily, Warner (I966) showed a significant increase in the population density of the flagellate Callimastix frontalis $\mathrm{I} \mathrm{h}$ after the sheep were fed. The population density then dropped to a minimum 8 to $10 \mathrm{~h}$ after feeding, after which little change occurred until the sheep were fed again. Samples of rumen fluid were taken immediately before feeding and at hourly intervals afterwards. The ratio of the maximum to the minimum population density was usually not more than 25 , but this is unusually high for rumen organisms where it normally fluctuates between 2 and 4 over $24 \mathrm{~h}$. Since 2 to 5 divisions of callimastix within $\mathrm{I} h$ followed by a rapid death of the majority of organisms (which would be one way of explaining the rapid population density fluctuation) was unlikely, it was suggested that the organism underwent sequestration on the rumen wall and entered the rumen liquor by chemotactic response to an acetone-soluble component in the diet soon after the animal was fed. It was also suggested that the organisms returned to the rumen wall afterwards in response to another chemical stimulus, perhaps a compound diffusing across the rumen wall from the blood system. As yet no large-scale active migration of the organisms of any kind within the rumen has been proved, although the stratification of food material may result in a passive redistribution of certain bacteria (e.g. the cellulolytic bacteria) which are physically attached to the particles (Hungate, I966). The problem of whether or not $C$. frontalis does migrate to and from the rumen wall is therefore of considerable interest, bearing in mind the continual mixing which occurs within the rumen and the relatively large distances involved.

\section{METHODS}

The sheep used were Clun Forests, fed daily a diet of $800 \mathrm{~g}$ hay chaff and $200 \mathrm{~g}$ crushed oats. Those used for in vivo population studies of $C$. frontalis were fitted with permanent rumen cannulae.

Single samples of rumen fluid were obtained by withdrawing io to $15 \mathrm{ml}$ of fluid by oral suction into a $\mathrm{I} \mathrm{cm}$ diam glass tube inserted through the cannula; samples were taken from random positions within the rumen until about $250 \mathrm{ml}$ of rumen fluid had been obtained, when this was mixed and subsampled. Each subsample was then strained through one layer of muslin to remove large particulate material. Samples were withdrawn from uncannulated sheep by stomach tube. The number of callimastix in any sample was estimated by counting microscopically all the callimastix in $0.01 \mathrm{ml}$ of the sample. Callimastix frontalis is morphologically distinct (Braune, 1914) from the other flagellate protozoa, with characteristic movements, and it is easily counted.

For experiments to determine if the callimastix was associated with the rumen, reticulum 
or omasum wall, 20 samples of rumen wall, eight of reticulum wall and two of omasum wall, each about $\mathrm{I} \mathrm{cm}$ square, were taken randomly from the organs of three freshly killed sheep which had not been fed for $24 \mathrm{~h}$, whose rumen fluid had been sampled before death and again immediately after death. These tissue samples were maintained at $39{ }^{\circ} \mathrm{C}$ and washed gently by hand in centrifuged rumen fluid (supernatant after $20000 \mathrm{~g}$ for $\mathrm{I} \mathrm{h}$ at $5^{\circ} \mathrm{C}$ ) to remove food particles. The portions of organ wall were then incubated under $\mathrm{CO}_{2}$ at $39{ }^{\circ} \mathrm{C}$ in $5 \mathrm{ml}$ centrifuged rumen fluid in the presence and absence of extract of I $\mathrm{g}$ dry wt of oats. Samples were taken after 10,20 and $30 \mathrm{~min}$ to see if callimastix were present in the supernatant. The oats extract was prepared as follows: $200 \mathrm{~g}$ of crushed oats were covered with $80 \%(\mathrm{v} / \mathrm{v})$ aqueous acetone and stirred by hand for I $\mathrm{h}$. The oats were filtered off, and the acetone removed by distillation under reduced pressure at below $40{ }^{\circ} \mathrm{C}$. The capacity of the extract to induce population changes of callimastix was determined by adding the extract from $200 \mathrm{~g}$ of oats to the rumen of a sheep which had not been fed for $24 \mathrm{~h}$, and estimating the callimastix population density before and $20 \mathrm{~min}$ after the addition of extract. An increase in population density greater than fivefold was taken to mean that the extract was active.

Incubation in vitro was performed using $\mathrm{I} \cdot 0 \mathrm{ml}$ of rumen fluid collected $24 \mathrm{~h}$ after feeding with and without the addition of $0^{\circ} \mathrm{I} \mathrm{ml}$ of active extract (equivalent to extract from $\mathrm{I} \cdot \mathrm{O} \mathrm{g}$ dry wt of oats).

Rumen fluid fractionation was performed as follows. Rumen fluid was strained through one layer of muslin. This did not allow the passage of particles of over $550 \mu \mathrm{m}$ diam. The filtrate was centrifuged at $1000 \mathrm{~g}$ for $15 \mathrm{~min}$ at room temperature $\left(20{ }^{\circ} \mathrm{C}\right)$ to remove protozoa and large particulate material. The supernatant fluid was centrifuged at $20000 \mathrm{~g}$ for $30 \mathrm{~min}$ at $5{ }^{\circ} \mathrm{C}$ to remove bacteria and small food particles. The $1000 \mathrm{~g}$ sediment was washed twice by centrifuging in $20000 \mathrm{~g}$ supernatant fluid, and resuspended in that fraction.

\section{RESULTS AND DISCUSSION}

Rumen fluid samples taken every $5 \mathrm{~min}$ after the onset of feeding revealed that the maximum population density of $C$. frontalis occurred within 15 to $30 \mathrm{~min}$ (Fig. 1). The maximum concentration occurred considerably earlier than detected by Warner (1966) although he sampled the rumen at hourly intervals and therefore missed the peak population density. The ratio of the population density maximum to the pre-feeding population density varied between 15 and 296 . The average ratio given by the results of 40 experiments using five different sheep was 47 . If allowances were made for the dilution of the rumen fluid over the feeding period, then the increase in population would be larger. Considerable variability was shown between different sheep and from day to day with the same sheep. Since it is very unlikely that callimastix divide an average of six times (necessary to explain the population increase in terms of binary fission of the organisms) within 15 min followed by a rapid death, there must be some other cause.

The ratio of population densities produced in vivo by the oats extract was 197, 20 min after the addition of the extract to the rumen. No callimastix was observed in vitro in the washings of the samples of rumen, reticulum and omasum walls, and no callimastix could be attracted from these samples by incubation in the presence of the extract of oats. The extract was active in vivo, and would therefore have been expected to have been capable of attracting the organisms from the tissue samples should they have been present when incubated in vitro. There was no increase in the concentration of callimastix in the rumen fluid immediately after the death of the sheep and the sampling of the organs. 


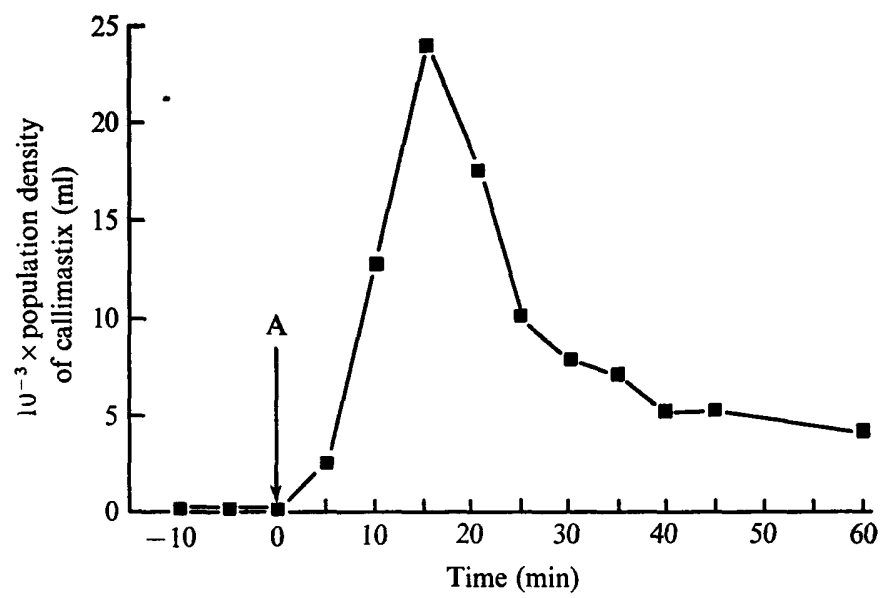

Fig. I. Population density curve for Callimastix frontalis over the feeding period of four sheep. The sheep were fed at point A. Samples of rumen fluid were taken at $5 \mathrm{~min}$ intervals and filtered through one layer of muslin, and the callimastix present in $0.01 \mathrm{ml}$ of the sample counted microscopically. The callimastix in three portions of each sample were counted, and the mean population density calculated. Means for the four sheep are plotted.

Samples of rumen fluid taken from the immediate vicinity of the rumen wall in vivo did not contain more callimastix than was found in samples taken from other positions within the rumen. The population density of the organisms did not change significantly with the depth of sampling within the rumen and was therefore independent of the stratification of food material which occurs.

These results indicate that sequestration on the rumen wall, as suggested by Warner (1966), does not occur. Therefore attempts were made to induce population changes of callimastix in vitro using the oats extract. The addition of the extract from $\mathrm{I} g$ dry $\mathrm{wt}$ of oats to each $\mathrm{ml}$ of fresh strained rumen fluid increased the concentration of callimastix 44-fold. The rumen fluid was fractionated further, and each fraction was tested for the generation of a concentration increase after the addition of the oats extract (extract of $\mathrm{I} \cdot \mathrm{g}$ dry $\mathrm{wt}$ of oats $/ \mathrm{ml}$ of rumen fluid). The results were as follows $\left(10^{-2} \times\right.$ population density of callimastix $/ \mathrm{ml}$, before and $20 \mathrm{~min}$ after the addition of the oats extract, respectively; mean of four experiments): whole strained rumen fluid $(6,268) ; 1000 g$ supernatant $(0,0)$; I000 $\mathrm{g}$ sediment $(4,224)$; 1000 to $20000 \mathrm{~g}$ sediment $(0,0) ; 20000 \mathrm{~g}$ supernatant $(0,0)$. This clearly shows that the callimastix are associated with the larger particulate fraction of the rumen fluid, containing the ciliate protozoa and larger food particles. The possibility of sequestration of the organisms on the rumen wall, with subsequent migration into the rumen after the host animal has fed and return to the rumen wall later, is therefore ruled out. It seems likely that either the organisms are secreted amongst the food material or else their life cycle involves a multiple reproduction phase which is associated with the large particulate fraction of the rumen fluid. In either case, the situation is novel for a rumen organism.

I thank Mrs M. Fordham and Miss A. L. Lowings for excellent technical assistance. 


\section{REFERENCES}

BRAUNE, R. (I9I4). Untersuchungen über die im Wiederkäuermagen vorkommenden Protozoen. Archiv für Protistenkunde 32, 1 I I-I 70.

Hungate, R. E. (1966). The Rumen and Its Microbes. New York: Academic Press.

WARNER, A. C. I. (1966). Diurnal changes in the concentrations of micro-organisms in the rumens of sheep fed limited diets once daily. Journal of General Microbiology 45, 21 3-235. 